\title{
Quand la favela devient 'comunidade' : nomination et contrôle social
}

When the Favela Becomes 'Comunidade': Designative Innovation and Social Control

\section{Patrick Dahlet}

\section{(2) OpenEdition}

\section{Journals}

Édition électronique

URL : http://journals.openedition.org/aad/2256

DOI : 10.4000/aad.2256

ISSN : $1565-8961$

Éditeur

Université de Tel-Aviv

Référence électronique

Patrick Dahlet, «Quand la favela devient 'comunidade' : nomination et contrôle social », Argumentation et Analyse du Discours [En ligne], 17 | 2016, mis en ligne le 15 octobre 2016, consulté le 23 septembre 2019. URL : http://journals.openedition.org/aad/2256 ; DOI : 10.4000/aad.2256

Ce document a été généré automatiquement le 23 septembre 2019.

\section{c) (i)}

Argumentation \& analyse du discours est mis à disposition selon les termes de la licence Creative Commons Attribution - Pas d'Utilisation Commerciale - Pas de Modification 4.0 International. 


\title{
Quand la favela devient 'comunidade ': nomination et contrôle social
}

\author{
When the Favela Becomes 'Comunidade': Designative Innovation and Social \\ Control
}

Patrick Dahlet

\section{Introduction}

1 Alors que jusqu'à une dizaine d'années encore, les occurrences attestées du terme comunidade(communauté) pour désigner la réalité urbaine et sociale de la favela étaient tout à fait épisodiques, voire quasi inexistantes, l'intensité de sa mise en circulation et de sa reprise par les discours institutionnels et médiatiques ont imposé depuis le terme en contexte brésilien comme mode d'appréhension et de catégorisation privilégié du périmètre géographique, fonctionnel et culturel de la favela.

Une double dynamique semble à l'oeuvre dans cette institution désignative. L'une, d'ordre cognitivo-sémantique, joue sur les disponibilités lexicales possibles du mot comunidade. Contre les amalgames discriminants qui façonnent les représentations de la favela, historiquement assimilée à un foyer d'infection et, plus récemment, de violence criminelle, elle vise à installer un modèle de reconceptualisation positive où l'emporterait le sens de l'entraide et de l'hospitalité. L'autre, de nature énonciativodiscursive, renvoie à la structuration et à la stabilisation d'un syntagme nominal, pacificação da comunidade, qui fait de la communauté la figure incontournable d'une pacification potentielle dont l'exécution est dévolue à des détachements de la police militaire, les UPP (Unités de Police Pacificatrice).

Il faut relever que ce corps de police a été spécialement créé à cette fin au moment même du processus de recatégorisation de la favela par le terme comunidade. Par ailleurs, les interventions des UPP, initiées sous cette forme à Rio en décembre 2007, se sont depuis multipliées et ont induit, en mai 2015, le dépôt d'un projet de loi ( $n^{\circ} 370$ / 
11) à la Chambre des députés en vue d'étendre le principe de la pacification à l'ensemble du territoire national.

4 Il ressort de cette triple coïncidence (réinscription de la favela dans le registre sémantique d'une comunidade, objectivation de ce registre dans la formule pacificação da comunidadeet contrôle policier d'un nouveau type) que la reconfiguration de la favela comme communauté ne fait pas seulement tomber dans le discours commun la formule d'un contrôle social et policier spécifique; elle apporte aussi la possibilité de sanctionner la réalité communautaire qu'elle invente par l'établissement d'une paix armée.

5 Comme on le verra, le parcours discursif du mot comunidades'avère à cet égard, en apparence en tout cas, on ne peut plus paradoxal, voire logiquement et sémantiquement contradictoire. En effet, il en vient à assembler dans une même formule et au sein des mêmes cadres énonciatifs, l'idéalisation d'un foyer commun, auto-régulé par le partage de proximité, d'une part, et la diabolisation de ce même foyer qui le rend justiciable d'un pouvoir discrétionnaire externe, d'autre part.

6 C'est l'administration de ce paradoxe par le terme comunidade et ses enjeux sociodiscursifs que l'on se propose d'étudier. Il s'agit en l'occurrence de comprendre comment le nouvel usage du terme concilie dans une même politique de (re)signification positive de la favela, comme secteur du réel urbain, l'annonce d'une mutation positive endogène, et l'énonciation d'une dénivellation discursive qui prend la prédiction de cette valeur à rebours pour faire de la coercition exogène une étape supérieure et englobante de cette mutation. Dans cette optique, l'hypothèse dont je pars est restreinte aux facteurs lexico-énonciatifs détectés et prend le risque de la ponction qu'elle opère dans une (dis)continuité multidimensionnelle non seulement linguistique et dialogique, mais aussi psychosociale et politique.

7 A partir du postulat selon lequel toute sélection désignative modélise le monde et intervient sur la représentation ainsi construite, on peut soutenir que l'activation simultanée de la substitution de favela par comunidadeet la formule pacificação da comunidade, qui l'inscrit explicitement dans un système de citations dialogiques, instituent un référent social inédit. J'entends par là que la singularité du référent produit par ce changement désignatif correspond, assurément, au pôle positif que le terme comunidadeveut opposer à la symbolisation globalement négative de la favela comme espace urbain indésirable. Mais, au-delà de ce programme de sens positif, l'articulation de cette conversion positive à l'ambivalence d'un ethos pacificateur cristallise une constellation inédite. En effet, au fil de leur entremêlement énonciatif, les termes de comunidade et pacificação apparaissent comme les composantes distinctes d'un même discours d'appropriation pour et contre doublement et indissociablement autorisé : d'un côté par l'amplitude, voire le flottement, des traits sémantiques - du lien humain à l'extension territoriale - qui caractérisent le mot comunidadecomme item lexical d'une langue, et de l'autre côté, par l'engagement du terme dans des pratiques énonciatives contraintes par la prédiction sémantique de ses valeurs, mais qui redistribuent son sens en fonction de critères d'une autre portée, en l'occurrence ici de nouvelles stratégies de développement territorial et de reconquête de l'espace urbain par l'action publique.

8 Dans cette perspective, après avoir situé mon propos dans le cadre conceptuel qui l'inspire et souligné la propagation du mot comunidadecomme matrice d'un mode de conceptualisation positif du monde de la favela, je repérerai dans un premier temps les 
(dé)compositions sémantiques du terme en m'arrêtant sur son régime lexicographique (qui est aussi, au moins partiellement, celui du sens commun) et j'éclairerai les enjeux de la ressource d'intelligibilité et d'emplois qu'elles constituent en invitant à penser l'objet favela autrement que par le prisme de la contamination ou de la violence : en l'occurrence comme soudé à la qualité primordiale d'être par la grâce de sa nouvelle désignation comme comunidade, ni plus ni moins que le lieu commun de ses ressortissants. Dans un deuxième temps, j'examinerai comment le processus de production politico-médiatique joue sur l'élasticité du sémantisme de l'item comunidade en langue et delivre ainsi une formule qui, en agglutinant comunidade et pacificação, construit la favela-communauté comme espace simultanément menaçant et menacé.

\section{Contre le stigmate de la favela, la propagation de la comunidade}

9 Avant d'en venir à la dynamique socio-énonciative déclenchée par le terme comunidade, j'esquisserai le cadre conceptuel de la proposition. Entendant le discours comme une réalité, celle d'un processus énonciatif de fabrication du monde qui institue, dans leur (in)consistance propre, «nos univers de discours » (Foucault 1994 [1968] : 675) et/ou un « monde de l'énonciation » (Benveniste 1974 [1969] : 64), ma démarche est régie par un double postulat.

10 Le premier, de nature épistémologique, implique qu'il n'y a pas d'un côté le discours ordonnateur de l'évènement et fabriquant du monde, et de l'autre l'hallucination de la réalité effective de la chose dans le nom qui l'habite. Il y a, indissociablement, le discours qui fait le monde dans l'univers de ses mots, et le sujet tenaillé par le désir de croire que ses mots disent bien la chose comme elle est. On retrouve ici la conception fondamentale de Benveniste, que le "langage [...] instaure une réalité imaginaire» (Benveniste 1966 : 25), ce qui peut s'entendre comme signifiant à la fois qu'il n'y a de réalité qui ne soit "produite en réalité dans et par l'énonciation » (Benveniste 1974 : 83) et que la force de cette prévalence linguistique tient irréductiblement aussi à l'illusion que nous partageons, du pouvoir germinatif de ses mots. Par suite, c'est tout acte de nomination qui constitue à part entière ses références dans ce jeu entre le pouvoir et la charge de réalité dont chaque sujet, pour son compte, le crédite. C'est bien ce qu'établit Searle en définissant les deux conditions de construction des «objets sociaux » par le langage :

Pour qu'un fait soit dépendant du langage, il suffit que deux conditions soient remplies. Il faut, en premier lieu, que des représentations mentales [...] soient en partie constitutives du fait; il faut, en second lieu, que les représentations en question soient dépendantes du langage (Searle $1998: 87$ ).

11 Le régime fondamental de fonctionnement de toute nomination est celui de cette dialectique entre la fabrication d'un simulacre du réel et le songe nécessaire qu'il ne s'agit pas là d'un simulacre, mais bien d'une matérialisation de la chose elle-même, garante de sa vérité et réalité. Si on reconnait la portée de cette dialectique, il faut alors aussi admettre, bien qu'il s'agisse d'une affabulation, que l'idée que le mot traduit la chose tient un rôle essentiel, au sens propre du terme, dans la puissance des déterminations venues du discours, faisant ainsi toujours peu ou prou de l'acte de nomination une ontologie de l'être. Quel que soit son degré d'opacité, tout discours est toujours ontologiquement concerné par la charge de réalité que ses protagonistes - 
chacun(e) d'entre nous - lui attribuent, ce " prédicat de réalité » dont est créditée toute assertion dans la définition qu'en donne Benveniste, en reliant toute assertion à l'économie implicite de l'articulation d'un «"cela est!" qui relie l'agencement linguistique au système de la réalité " (Dahlet 1996: 106-107). La substitution de comunidadeà favela trouve dans le crédit de réalité accordé à la relecture ainsi faite de l'entité favela, l'un de ses motifs essentiels, là aussi dans tous les sens du terme.

Quant au deuxième postulat, il renvoie à l'idée que toute opération de désignation terme que je retiens, en référence à Culioli (1990: 83-90), tout en comprenant les réserves de Siblot (2001: 190-193) sur l'extension non contrôlée des acceptions de dénomination et désignation - correspond au choix (in)volontaire d'un terme, au sein d'un «paradigme désignationnel» (Mortureux 1984; 1993), sans que les termes non sélectionnés ne cessent de travailler, en leur absence même, le terme retenu pour construire l'objet.

13 Étant une au sein d'un faisceau de possibles, toute désignation se fraie un chemin entre et/ou contre eux, et construit ainsi toujours son objet sous un certain point de vue sémiotiquement parlant, symboliquement spécifiant, dialogiquement profilé (Bakhtine 1977 [1929]) et incrusté dans une «mémoire discursive " (Courtine 1981, Moirand 2007).

Le charme du possible réel que la propulsion du mot comunidadeoppose au stigmate de la favela s'inscrit dans le droit fil de ce double postulat.

De fait, la récente généralisation du terme comunidade comme désignation de la favela dans les pratiques discursives brésiliennes est incontestable. Sauf indication contraire, tous les exemples qui suivent sont extraits de la presse brésilienne en ligne (Folha de São Paulo, Estadaõ de São Paulo et TV Globo) de la période 2012-2015, (donnés directement dans ma traduction française) avec tous les désignants de la favela surlignés en italiques, et accessibles en brésilien par les liens mentionnés.

16 Ainsi, alors que les occurrences du mot comunidade dans cette fonction sont rares durant tout le vingtième siècle (face, par exemple, à la centaine de chansons recensées sur le thème de la favela, avant les années 2000, je n'en ai trouvé aucune qui la thématise comme comunidade, la chanson religieuse paraissant avoir le quasi monopole du terme), le terme apparait aujourd'hui massivement, isolément et/ou en alternance avec favela, dans les discours qui l'évoquent, à l'instar de celui-ci :

(1) Je continue à habiter dans la favela [....] J'ai créé le Musée de la Favela en 2009 [...].

Aujourd'hui c'est une référence territoriale au Brésil et dans le monde au sein des comunidades [...] J'ai accédé à une position [...] de leader dans la favela [...] mais déterminé à me battre toujours pour les causes de la comunidade [...] et à réaliser des choses dans la comunidade. [...] Je crois beaucoup au pouvoir [...] de ma trajectoire pour la comunidade [...] une comunidade se transforme au cours des années [...] (Interview avec le graffitiste ACME, hebdomadaire Brasil De Fato 2-8/7/15, p. 10-11).

L'alternance systématique comunidade / favela dans ce seul exemple suffit à démontrer le rôle majeur attribué au terme comunidade dans la modification du choeur social autour de la favela.

Il n'existe pas à ma connaissance d'analyse linguistique de la trajectoire discursive du mot favela, et encore moins de celle, trop récente, de sa reprise par comunidade. Mais la chronique des prises de position sur l'espace indésirable qu'aurait inauguré la première désignation d'une favela - o Morro da Favella / le Morne de la Favella, orthographié originellement avec deux « $1 »)$, - à la fin du dix-neuvième siècle à Rio, et l'abondante 
littérature sociologique et anthropologique consacrée à l'histoire et aux représentations de la favela (notamment Bretas 1997, Alvito et Zaluar 1998, Silva 2005, Valladares 2005, Machado da Silva 2008) convergent pour autoriser à rattacher la propagation du terme comunidade comme denotatum de la favela, à une politique de (re)signification visant à crédibiliser l'engagement institutionnel d'urbaniser les favelas et de veiller à l'intégration sociale de leurs populations, sans cesse réaffirmé depuis la redémocratisation du pays (1984-1990).

Une telle politique de (re)signification pose par définition la fonction sémantique spécifique de l'extension de la désignation de comunidade pour référer à la favela en contrepoint à la sédimentation stéréotypique du mot favela lui-même, obéissant à une topique du type " ce qui est sans qualités formelles visibles, est menaçant ».

Double menace à vrai dire, pathogène et sociale, dont l'édification discursive s'ouvre avec le temps de la favela lui-même, "foyer de déserteurs, voleurs et hommes de troupes » (Jornal do Brasil 1900, Bretas $1997:$ 75) et se poursuit jusqu'à aujourd'hui aux heures de grandes écoutes télévisées - "Vous vous imaginez! ma nièce perdue dans une favela, dans ce repaire de pauvres et de bandits »; " Vous ne savez pas ce qu'est la favela! Je ne peux pas revenir dans ce lieu ! Là-bas c'est la barbarie, c'est sauvage, moi je veux être classe moyenne " (Telenovela de la Chaîne Globo, 19/10/15 et 18.11.15, 21h15) -, relayée par une accumulation de paroles d'autorité, au nombre desquelles, celle-ci, de l'architecte français Alfred Agache, dans le projet de remodélisation de la ville de Rio, alors capitale du pays, qu'il a élaboré à l'invitation de son maire à la fin des années 1920 :

les favellas constituent un danger permanent d'incendies et d'infections épidémiques pour tous les quartiers au sein desquels elles s'infiltrent. Sa lèpre souille les plages et les quartiers plus gracieusement dotés par la nature [...] (Agache 1930: 190 - italiques dans le texte ; je traduis du brésilien). radicalement ceux qui sont de la favela et ceux qui sont de l'extérieur, je ferai simplement observer que les favelas se trouvent aujourd'hui définies par les programmes de réurbanisation comme des «zones d'occupation précaires, irrégulières, clandestines, à risques » (Prefeitura de São Paulo, Plano Diretor Estratégico, 2014, art. 18) et/ou comme des "agglomérats infranormaux caractérisés par l'absence de titres de propriété » (Instituto Brasileiro de Geografia e Estatística, Censo / Recensement 2010 : 3). Dans la vision des pouvoirs publics, la favela demeure ainsi un objet urbain déviant et porteur de virtualités négatives, toutes virtualités que la promotion du terme comunidade - qui, significativement, n'apparaît pas dans les plans d'aménagements urbains - prétend précisément marquer du sceau de la concorde primordiale.

\section{2. (Dé)compositions sémantiques : l'avoir en commun lesté d'extranéité}

Si l'on admet que toute modélisation lexicographique fixe dans sa description les schèmes du sens commun, la consultation des dictionnaires brésiliens de langue apporte des éléments précieux à la compréhension de l'affectation de comunidadeà cette fonction pivot de resémantisation positive de la favela. Dans le Dictionário Da Língua Portuguesa (Muniz et al. 2010), la description du terme comunidadearticule six entrées : 
1 Qualité ou état de ce qui est commun 2 Tout groupe social dont les membres habitent une région déterminée, ont le même gouvernement et sont culturellement et historiquement apparentés $3^{\circ}$ Tout ensemble de population considéré comme un tout, en vertu d'aspects géographiques, économiques et/ou culturels communs $4^{\circ}$ Dans une formation sociale complexe, groupe d'individus considéré dans ses caractéristiques spécifiques et individualisantes $5^{\circ}$ Ensemble d'individus qui vivent en commun $6^{\circ} \mathrm{Groupe}$ de personnes qui partagent une même croyance ou idée.

foncièrement, du qualitatif " pur » - la qualité ou état de ce qui est commun - qui ne définit une collectivité humaine par aucune autre propriété que celle d'être semblable à elle-même, autrement dit construite et entretenue à partir du rapport d'inclusion réciproque des individus qui la forment. En ce sens, la comunidadec'est la désignation d'une qualité essentielle, celle d'un "être ensemble», dérivé de la formulation d'un «avoir en commun ». Et cela comme par enchantement, puisqu'on a bien affaire à une nominalisation déadjectivale qui à la fois ne trace pas son procès et impose le présupposé d'existence que lui confère sa détermination par l'article défini. C'est ce quelque chose d'unique que conforte l'identification de la comunidade, quoi qu'il en soit de son mode d'existence, par l'exclusivité d'un nom propre : «La comunidade Cidade de Deus a été rendue mondialement célèbre par le film homonyme de Fernando Meirelles [...] La comunidade Santa Marta est aussi très souvent appelée Dona Marta ${ }^{1}$ ». Qand elle renvoie ainsi sans reste à de l'«être en commun» sa désignation comme comunidadedénote nécessairement la favela, à des années lumière du stigmate, à travers une communion dont elle reconstitue et célèbre la mémoire. Mais c'est toujours avec le correctif possible de la suite des acceptions lexicographiques du terme.

Sans entrer dans le détail des cinq entrées supplémentaires de la définition considérée, on relèvera que, au-delà d'une variable quantitative - " avoir en commun » à l'échelle d'un groupe $(2,4,5,6)$ ou d'une collectivité plus vaste (3) - toutes spécifient en effet, d'une manière ou d'une autre, un critère de délimitation et de reconnaissance du fait en question comme étant de nature communautaire. Le spectre des critères invoqués est large (géographiques en 2 et 3, économiques et culturels en 3 , sociaux en 4 , éthiques et idéologiques en 5 et 6 ) et l'itération discursive de comunidadele démarque par définition en permanence, à l'instar des énoncés ci-dessous :

(3) Dans la comunidade du quartier de Barro Branco - à moins de $10 \mathrm{~km} \mathrm{du}$ Pelourinho, un des points touristiques de la capitale bahianaise -, Gabriel, 8 ans, pleure de désespoir [...] (hebdomadaire Brasil De Fato 8-14/10/2015, n658, p. 8).

(4) La comunidade uspienne réclame des changements qui légitiment l'USP [Université de São Paulo] comme université critique, démocratique et publique ${ }^{2}$.

(5) Au Brésil, [Jean-Paul 2] a réprimé la Théologie de la Libération et restreint la vigueur des Comunidades Écclésiastiques de Base ${ }^{3}$. 
(6) L'ouvrage met en scène des personnages typiquement cariocas : classe moyenne cosmopolite, population de comunidades démunies et riches dans leurs châteaux ${ }^{4}$.

(7) Les droits des comunidades indigènes et afrodescendantes, par exemple, doivent être encore plus protégés qu'ils le sont à l'heure actuelle ${ }^{5}$.

Par le biais d'une expansion syntaxique, la qualité communautaire essentielle est plongée dans des univers discursifs distincts : géographiques (3), universitaires (4), religieux (5) et sociaux (6 et 7). Mais, du même coup, c'est le principe de sélection en question qui détermine indissociablement la valeur référentielle de comunidadeet non plus la seule notion de mise en commun dont le terme peut être porteur.

Et la qualité essentielle d'avoir quelque chose en commun induit une interprétation toute différente lorsque la référence de ce quelque chose renvoie à une catégorisation identitaire, et plus particulièrement juridico-administrative ou ethnico-sociale, parce que, dans ce cas, la valorisation de la primauté de l'union s'efface derrière l'homogénéisation par les caractéristiques de l'appartenance dont elle est issue.

8 Dans ce cas, ce n'est pas le schème communautaire qui vaut en soi, mais le principe de sélection constitutif du lien communautaire. Ce n'est plus le fait communautaire qui est en lui-même significatif, mais l'appariement des membres de la communauté et du principe de sélection en question. Et l'on passe alors ainsi insensiblement de la référence au fait qu'une agrégation d'individus est positivée par son essence communautaire, au critère qui les identifie comme relevant de cette communauté-là, et pas d'une autre, et les oppose au Nous qui l'énonce.

(8) Le public a pu assister à des défilés de mode africaine, shows musicaux et vidéos de tribus et comunidades afrodescendantes. ${ }^{6}$

(9) Region multiéthnique, avec des comunidades indigènes, paysannes et afrodescendantes, le département de Cauca, au sud de la Colombie, est un des bastions des Farc. ${ }^{7}$

Le terme comunidadeparaît bien fonctionner ici comme un marqueur ethnotypique de l'Autre au coeur même de la ressemblance qu'il condense - entre les membres du rassemblement en question et l'ensemble du tissu social lui-même, puisque celui-ci est à la fois tramé d'innombrables structures communautaires de tout type et se donne luimême comme comunidade(trans)nationale.

$\mathrm{Au}$ bout de ce parcours lexico-sémantique, je dirais que la plasticité et le chevauchement des acceptions du terme autorisent la manipulation des critères et de l'ordonnancement de leurs partages. De fait, la qualité substancielle d'un « avoir / être en commun ", primordiale dans la définition du mot, est circonscrite comme dispositif de partition territoriale et/ou identitaire, et consécutivement argument d'(il)légitimation possible de cette identité comme altérité hostile et hostilisée. La glose de comunidade en termes territoriaux et/ou identitaires est latente. Ces traits sont constitutifs de la (dé)composition sémantique du terme, mais à la manière d'un "signifié de puissance», selon la terminologie guillaumienne. Autrement dit, ils relèvent d'un dicible en langue, plus ou moins (in)conscient, duquel tout énonciateur, en position de le faire, peut se réclamer pour classifier une communauté, tout en paraissant se rendre à l'évidence de son sens qualitatif englobant. 


\section{La comunidade fondue dans sa pacificação}

31 Telle qu'on l'a décrite, la dynamique sémantique du mot comunidade lie l'authentification d'une qualité essentielle du lien social et la démarcation potentielle entre intérieur et extérieur constitutifs de sa spatialisation.

C'est dans ce double mouvement sémantique que prennent place simultanément la systématisation du terme, à partir de 2005, dans le rôle de désignant de la favela, et la mise en circulation du syntagme pacificação da comunidade. Ce dernier acquiert très vite, sous l'effet de la densité de ses reprises médiatiques, l'autonomie sémanticoréférentielle d'une formule, que l'on entend, à la suite de Krieg-Planque (2009) comme forme de figement de "quelque chose pour tous à un moment donné » (ibid. 95), qui objective un référent social inédit en stabilisant en un tout de façon sélective, et donc potentiellement conflictuelle, des significations à l'oeuvre dans chacune de ses parties. Au-delà des innombrables occurrences médiatiques de la formule dans les dernières années, le fait notoire est que, contrairement à d'autres désignations impliquant la notion de favela, celle-ci s'est immédiatement traduite par la création et l'installation dans les comunidades, dès le début de l'année 2008 d'un dispositif militaro-policier spécifique: les Unidades de Policia Pacificadoras (Unités de police pacificatrices,UPPs, selon le sigle officiel).

Qu'est-ce qui fait de cette formule le catalyseur, simultanément parlant et agissant, de ce réel-là? Pour tenter de mettre en évidence les (dé)tours linguistiques qui la conditionnent et le sens social qui y prend corps, je partirai des exemples médiatiques suivants, qui offrent un condensé (v. les trois modes de régulation explicités ci-dessous) des mécanismes de production et de légitimation de la formule en question :

(10) La pacificação des comunidades [...] de la zone sud de Rio, a commencé [...] ce dimanche [...] Les comunidades sont déjà au pouvoir de l'État. [...] Après la reconquête du territoire jusque-là dominé par des criminels, les comunidades recevront sous peu la 19e UPP de l'État. [...] Avec la pacificação, les 72458 habitants de Rocinha [...], outre la population qui vit à l'entour des comunidades, seront bénéficiaires ${ }^{8}$.

(11) L'escalade de la violence criminelle dans les comunidades populaires est due principalement à l'incapacité de l'État de garantir sa présence permanente et souveraine dans les favelas, permettant que des groupes armés construisent un processus autoritaire de régulation du territoire ${ }^{9}$.

(12) La pacificação achève sa cinquième année à Rio [...] Avec près de 6000 habitants, la comunidade de Santa Maria a été la pionnière de cette politique sécuritaire, dont l'objectif est d'expulser les groupes armés et d'offrir les services publics aux habitants. [...] Jusqu'à présent, la police n'osait mettre les pieds dans les favelas qu'à l'occasion d'incursions ponctuelles et, pour la première fois, elle a décidé de s'installer de forme permanente avec des détachements connus comme UPP ${ }^{10}$.

(13) La pacificação de favelas à Rio valorise les quartiers proches des comunidades et ouvre un nouveau marché pour les entreprises de construction et de viabilisation ${ }^{11}$.

(14) Les jeunes des comunidades soulignent les bénéfices de la pacificação ${ }^{12}$.

Sous l'éclairage de ces énoncés, le régime discursif de la formule, qui explique le pouvoir d'imposition de son réel, peut être reconstruit comme résultant de la composition entre eux de trois modes de régulation, liés respectivement au déploiement de la disparité sémantique du mot comunidade, à l'antériorité dialogique de la notion de pacificação dans le contexte brésilien et à l'effet d'évidence de son institution nominale. 
35 La première régulation, fondamentale, joue sur la dissociation de l'Avoir (en commun) et de l'Être (d'une certaine communauté territoriale et/ou éthnique) qu'on a cru pouvoir relever dans la description lexicographique du mot comunidade. On ne reviendra donc pas ici sur la consistance du phénomène, si ce n'est pour distinguer la signification de son exploitation discursive dans l'entour de la formule pacificação das comunidades.

36 À cet égard, l'observation des exemples suffit à mettre en évidence une répartition énonciative, voire un clivage, tout à fait remarquable des emplois du mot comunidade, jouant sur l'élasticité de son potentiel sémantique, telle que l'a fait ressortir l'approche de son régime lexicographique ( $\mathrm{v}$. plus haut), plus malléable, car distinct, de celui de favela.

37 De fait, alors que la pacificação, en tant que dispositif d'intervention militaire-policier, saisit comunidadedans le sens de territoire (cf. l'homogénéité du champ topique établi par la substitution de «territoire » à comunidadeen 10 et 11), ses effets sont en revanche référés, via la convocation de l'emploi identitaire de comunidade, non à l'espace mais aux personnes qui l'habitent, comme le dénote la collocation synonymique de comunidadeet "habitants de Rocinha » en (10) ou l'extraction des jeunes, qui décline comunidadecomme une collection de ressortissants en (14).

38 Symptomatique a contrario de cette division discursive, est la coïncidence énonciative des deux valeurs en (12), où le nom propre Santa Maria fait de comunidadele référent d'un territoire pendant que sa qualification comme " pionnière de cette politique sécuritaire » la configure comme fonds commun d'un ensemble déterminé d'individus, cette coalescence dotant apparemment la pacificação de la performativité consensuelle (l'occupation du territoire au service de ses habitants) à laquelle elle prétend dans l'imaginaire collectif.

39 Cette première régulation n'élimine évidemment pas, puisqu'elle est structurée en langue, la scène essentielle de la qualité de l'«avoir en commun » incluse dans le domaine de signification de comunidade. Cependant, en l'absentéisant, le filtrage discursif du sens de comunidadecomme territoire et/ou identité, autorise la pacificação à la fois à s'exercer (en matérialisant un espace de souveraineté et des sujets auxquels s'appliquer) et à se présenter simultanément comme redoutable pour les uns et prometteuse pour les autres. La comunidadeest ainsi rendue présente par l'éclipse d'un idéal communautaire qui ne continue pas moins à la travailler.

40 C'est en embrayant sur une telle coalescence hétérogène des sens du mot comunidade, que la formule pacificação das comunidades réussit ce tour de force (discursif) de constituer ce « quelque chose pour tous » qu'elle fabrique, comme quelque chose de plus et d'autre que le support d'une opération policière : la localisation et la loi d'une nécessité qui prennent corps et sens dans et par leur formulation discursive elle-même.

41 Toute formule convoque dans son processus d'hybridation sémantique la mémoire discursive qu'y incrustent ses termes. À cet égard, le deuxième mode de régulation enregistre le dialogisme spécifique du terme pacificação.

42 Il est clair, trop clair sans doute (v. ci-dessous l'effet de « déjà-là » de la nominalisation) que le terme pacificação inscrit l'expérience communautaire dans un contre-discours conduit par une logique de guerre, en référence à laquelle un territoire devient l'instrument d'un conflit entre des parties dont l'une cherche à soumettre l'autre par des moyens violents. Si c'est le trait sémantique /territoire/ qui justifie l'emboîtement, 
je dirais que celui-ci doit son succès médiatique comme formule à une double antériorité dialogique en contexte brésilien.

Premièrement, en devenant l'expansion d'une pacificação, la comunidadese met à exister en rapport avec l'épopée de l'unification nationale, incarnée dans l'imaginaire collectif par les campagnes de pacificação, ainsi nommées, menées au dix-neuvième siècle par le Duque de Caxias, qui l'ont fait reconnaître comme "patron de l'armée brésilienne » en 1962, et continuent à lui valoir régulièrement des hommages officiels à l'instar de celuici, du sénateur Romeo Tuma :

(15) Ce héros [...] Caxias a été essentiel dans la pacificação de diverses provinces qui se sont soulevées en armes. [...] Il a traité les prisonniers avec dignité et respect. [...] À trente neuf ans, il était déjà général et pacificador de trois provinces (Romeu Tuma 2003).

Deuxièmement, le point de vue guerrier construit par pacificação renvoie inévitablement le désignant comunidadeaux rebondissements d'une formule spécifique antérieure : celle de Batalha das favelas [Bataille des favelas], alternant avec Batalha de Rio de Janeiro, sous laquelle le quotidien Correio da Manhã a initié en 1948 une campagne pour résoudre le "problème des favelas " qui s'est multiplié monstrueusement , amenant 400.000 brésiliens à vivre 'une vie à part' [...] en marge de la comunidade " (Correio da Manhã 19/6/1948, p. 2 - et on aura relevé au passage que comunidade désigne significativement à l'époque tout et exclusivement le dehors de la comunidade / favela actuelle), formule qui elle-même se retrouve recyclée par l'intitulé Guerra do Rio que lance 59 ans plus tard, en 2007, le jornal o Globo pour rendre compte pendant deux mois de l'occupation militaire de l'ensemble des favelas de Rio, dénommé Complexo do Alemão.

Il s'agit encore de l'ocupação d'un Complexo, qui ne sera typifiée comme pacificação, corrélativement à la création des UPP, que l'année suivante, pour amortir rhétoriquement le choc de l'invasion au bénéfice d'un retour à la paix qui ne contredise pas le bien essentiel attaché à l'idée de communauté, sans pour autant disqualifier l'emploi de la violence étatique: c'est précisément au rétablissement et à la préservation de ce bien originaire que le mot pacificação voue la conquête guerrière qu'il revivifie.

46 Cette dissolution énonciative du schéma standard de l'affrontement repose par définition sur l'abstraction de son terme de départ que réalise l'opération de nominalisation (désormais NMS). C'est le troisième mode de régulation de la formule que l'on pointera.

47 On sait que l'opération de NMS, en vidant la prédication verbale correspondante de ses conditions d'énonciation (non repérage de l'énonciateur, de la localisation, des actants et de leur causalité), impose son univers sous la forme d'un préconstruit pensé indépendamment de ses circonstances de production (Pêcheux $1990: 193$ et 221) et qui " produit un effet d'évidence, de 'déjà-là' , de construit non dans et par le discours, mais dans le réel » (Sériot 1986: 25 - italiques originales). Si l'on observe en outre que dans tous les exemples cités, le marquage défini attribue à cette représentation déréférentialisée une dimension toujours vraie, susceptible d'être dite et redite comme telle par tout locuteur, on peut considérer que la formule a pacificação da comunidade(re)classifie l'action de pacifier, dans le cas d'une favela à affinités communautaires, comme littéralement « incontestable, parce qu'elle soustrait à toute 
contre-argumentation les termes de son argumentation, dans la mesure où ils se confondent avec un pré-construit invisible » (Dahlet $2015: 211$ ).

Mais la formule ne se contente pas d'exploiter l'aptitude de toute NMS généralisante à doter son objet d'une intouchable charge d'évidence. Elle montre l'assomption communautaire de la favela en train de s'accomplir visiblement dans le mouvement même du discours qui prône invisiblement la naturalité de sa pacificação.

Je veux dire par là que les contextes d'insertion de la formule pacificação da comunidadeont ceci de remarquable qu'ils génèrent et exposent à la vue la mutation qualitative anticipée par la substitution désignative de comunidadeà favela. De fait, en relisant les exemples, on s'aperçoit que l'impuissance de l'État typifie l'espace-objet comme favela (voir 11: «l'incapacité de l'État de garantir sa présence permanente et souveraine dans les favelas ") et que, a contrario, la présence réaffirmée de l'État à travers la pacificação, constitue ipso facto les favelas en comunidades (10: «Les comunidades sont déjà au pouvoir de l'État »). Mieux encore, ce sont les pôles mêmes de cette mutation énonciative interne que met en scène la formule, comme en témoigne, on ne peut plus nettement, la métamorphose de la favelaen comunidade(en 13), «La pacificação de favelas à Rio valorise les quartiers proches des comunidades ", et (en 12) «la comunidade de Santa Maria a été la pionnière de cette politique sécuritaire [...] Jusqu'à présent, la police n'osait mettre les pieds dans les favelas ». "Misérable miracle " peut-être, dirait Michaux, mais tour de force discursif en tout cas, d'une formule qui prouve dans son usage même la force de la reclassification désignative de la favelacomme comunidade. Une force qui, en désignant à neuf la favela, ne la convertit pas seulement en comunidade, mais y fait émerger et y reconnait de l'être attaché à une communauté humaine. En effet, comme le confirme l'un des exemples, dès lors qu'on souligne sans reste les bienfaits de la pacification, on n'est plus une subjectivité excentrique, identifiée par son appariement à la paire catégorielle « jeunes des favelas »etfrappée de discrédit par la typification sous-jacente de la menace de la favela et de ses effets de contamination. Qualifié tout uniment comme "jeunes ", on devient reconnaissable comme sujet d'une ressemblance, et consécutivement on n'est plus d'une favela mais on habite une comunidade.

51 L'évidence préconstruite de la formule pacificação da comunidadeest ainsi explicitement construite, par redistribution de ses composantes (la police et ses adversaires, la population et son territoire), au sein d'un mouvement argumentatif où se joue la transformation d'un discours au nom de l'État contre des factions criminelles, en discours au nom de tous pour un état communautaire nouveau post-pacificação.

52 En ce sens, parler de la pacificação das comunidades, c'est faire coïncider, sous l'effet de nos trois modes de régulation, la présupposition d'évidence et d'existence propre à toute NMS générique et la construction d'un récit sur les comunidades et les causes de leurs problèmes, qui fait de l'éradication d'une violence préexistante le motif de l'application de la dite pacificação aux dites comunidades et de la première une catégorie banalisée de l'action publique sur les secondes.

53 Et cela d'autant plus que l'intense médiatisation de la formule pacificação das comunidades a entraîné une revitalisation frappante de l'usage du terme pacificação. Jusque-là cantonné à l'évocation des campagnes militaires nationales du dix-neuvième siècle, le terme est régulièrement mobilisé aujourd'hui, parallèlement à son application à la réalité des comunidades, dans le discours politico-médiatique. On ne compte pas moins de 60 discours qui ont traité au sénat de la pacificação des relations 
institutionnelles et/ou internationales depuis $2008^{13}$, et la presse reprend à l'envi la forme verbale ou nominalisée du terme, comme outil de relecture de situations données comme construites par le conflit :

(16) « penser que, hors de la constitution et de la loi, on va réussir à pacificar le pays

[...] est une erreur grossière » a dit Cardoso [ministre de la justice ${ }^{14}$.

(17) Dilma [presidente de la république] a affirmé : [...] les coups d'État ne construisent pas l'harmonie et l'unité, ni la pacificação nécessaire aux avancées du pays $(8 / 12 / 15)^{15}$.

Sous le double coup de l'aura médiatique de la formule pacificação da comunidadeet de la banalisation du terme pacificação dans les discours sociaux, ce qui ne cesse alors de reculer, ce sont les limites de sa portée narrative, et consécutivement sociale. Le crescendo de l'usage de la formule va de pair avec celui de son espace de légitimité, comme le démontre cet autre titre de presse :

(18) Le nouveau recteur de l'USP [Université de São Paulo] aura pour mission de pacificar a comunidade ${ }^{16}$.

Alors même que la formule pacificação da comunidadese présente comme une catégorie administrativo-judiciaire de classement et de gestion spécifique de la favela, maintenant comunidade, elle devient une synecdoque de la régulation de toutes les temporalités de l'espace social, figure d'une rhétorique de la refondation de nouvelles attaches politiques et sociales, quand elles viennent à manquer.

Par là-même, elle n'est plus la formule d'un agir extraordinaire trouant l'ordre du quotidien par le bruit et la fureur, y compris de sa puissance de feu (il n'est pas de jours sans morts provoquées par la pacificação des favelas-comunidades), mais la référence à une normalité consensuelle qui en fait une option de gouvernement des mouvements sociaux et de correction des obstacles à la viabilité de la vie nationale en commun.

\section{Conclusion}

57 Contre l'idée que le discours procède de la réalité, c'est la réalité qui procède du discours, au fil de l'arborescence des transformations désignatives qui affectent ses points de vue, et des discontinuités dialogiques qui les apparient, de façon plus ou moins diffuse, à d'autres actes de (re)production de leurs termes. C'est du moins cette représentation de la force signifiante du discours, et de la désignation, en particulier, comme pivot de la fabrication de ce monde de discours, qui a frayé le chemin de cette approche du passage récent à la projection énonciative et à la notoriété médiatique du mot comunidade, dans le rôle de désignant de la favela en contexte brésilien.

58 À cet égard, j'espère avoir montré les mécanismes de la double fertilité symbolique attendue de ce passage, à savoir d'une part comment la vocation à la destruction du stigmate, attribuée à la socialisation du terme comunidade, a joué sur le cercle vertueux d'une communion de fondation condensée dans son sémantisme, et d'autre part comment l'intense réénonciation de ce terme au sein de la formule pacificação da comunidades'est elle-même autorisée des sèmes complémentaires de /territoire/ et / identité/ intégrés à son paradigme définitionnel, pour décliner la " naturalité » d'une réappropriation militaire-policière de la comunidade, resémantisée comme comunidade pacificada [communauté pacifiée], dès lors que ses collections d'indésirables ont été repoussées hors de ses limites, au nom du bien public : 
(19) Les fusillades entre factions criminelles et police [...] ont repris dans les comunidades pacificadas ${ }^{17}$.

(20) Deux personnes meurent à la suite de fusillades dans des comunidades pacificadas $^{18}$.

Le tourniquet discursif est à la fois paradoxal et impressionnant. Il est paradoxal parce qu'il présuppose maintenant que, sous l'effet de sa collocation avec pacificação, le pôle qui organise la signification du terme comunidaden'est pas la qualité d'un «avoir en commun ", qui a déclenché la promotion du terme, mais un détonateur guerrier. Et il est impressionnant parce que, en présentant sur fond de pacificação, la comunidade, affectée par des points d'hérésie, comme ne concordant pas avec l'idéal communautaire qu'est chargé de désigner le terme, la formule en génère une autre : celle de comunidade pacificada.

Tout se passe comme si la communauté pacifiée devenait désormais la figure ultime et première de la favela-comunidade, et qu'elle était restaurée dans sa qualité fondamentale d'humanité - celle-là même qui est radicalement à l'oeuvre dans le sémantisme de comunidade -, par la formule d'une pacificação, elle-même généralisable, comme mode de traitement de tout conflit, à la globalité du système social, laissant ouverte la question de savoir ce qui peut obliger une collectivité à se retrouver sous le sceau de ses vérités d'évidence, comme si c'étaient les siennes, et s'il y a quelque possiblité de sortir de leurs clous sans reconduire des origines fantasmées ou forgées pour toujours.

\section{BIBLIOGRAPHIE}

Agache, Alfred. 1930, Cidade do Rio de Janeiro extensão - remodelação - embelezamento (Rio de Janeiro : Prefeitura do Distrito Federal)

Althusser, Louis. 1976. «Idéologie et appareils idéologiques d'état. Notes pour une recherche», Positions (Paris: Editions sociales ), 67-125

Alvito, Marcos \& Alba Zaluar (éds). 2003. Um Século de Favela (3ème éd.), (Rio de Janeiro : FGV)

Amossy, Ruth. 2012 [2000]. L'argumentation dans le discours (Paris : Dunod)

Amossy, Ruth. 2014. Apologie de la polémique (Paris : PUF)

Bakhtine, Mikhail. 1977 [1929]. Le marxisme et la philosophie du langage (Paris : Minuit)

Benveniste, Émile, 1966 [1963]. Problèmes de linguistique générale, t.1 (Paris : Gallimard)

Benveniste. Émile. 1974 [1969/70]. Problèmes de linguistique générale, t.2 (Paris : Gallimard)

Bosredon, Bernard, Tamba, Irène \& Petit, Gérard (éds). 2001. Linguistique de la dénomination

(Cahiers de praxématique36)

Bretas, Marcos. 1997. Ordem na cidade: o cotidiano da autoridade policial no Rio de Janeiro, 1907-1930

(Rio de Janeiro: Rocco) 
Cassanas, Armelle et al. (éds). 2004. Dialogisme et nomination, Actes du IIIe colloque Jeunes chercheurs (Montpellier : Publications de l'Université Paul-Valéry)

Cislaru, Georgeta et al. (éds). 2007. L'acte de nommer. Une dynamique entre langue et discours (Paris : Presses Sorbonne Nouvelle)

Courtine, Jean-Jacques. 1981. «Quelques problèmes théoriques et méthodologiques en analyse du discours. À propos du discours communiste adressé aux chrétiens », Langages 62, 9-128

Culioli, Antoine. 1990. Pour une linguistique de l'énonciation, t. 1(Paris : Ophrys)

Dahlet, Patrick. 1996. « Benveniste et l'effusion énonciative de la langue ». Sémiotiques 10, 99-121

Dahlet, Patrick. 2015. «(Re)produzir o inquestionável : nominalização, generalização e naturalização no discurso neoliberal ", EID\&A - Revista Eletrônica de Estudos Integrados em Discurso e Argumentação 8, 205-220 [En ligne http://www.uesc.br/revistas/eidea/index.php? item=conteudo_sumario8.php]

Foucault, Michel. 1994 [1968]. « Réponse à une question », Dits et Ecrits, t. I (Paris : Gallimard), 673-695

Instituto Brasileiro de Geografia e Estatística. 2010. Censo / Recensement 2010 [En ligne : http:// censo2010.ibge.gov.br/]

Krieg-Planque, Alice. 2009. La notion de « formule » en analyse du discours. Cadre théorique et méthodologique (Besançon : Presses universitaires de Franche-Comté)

Machado da Silva, Luiz Antonio (éd.). 2008. Vida sob cerco: violência e rotina nas favelas do Rio de Janeiro (Rio de Janeiro : Faperj/Nova Fronteira)

Moirand, Sophie. 2007. Les discours de la presse quotidienne (Observer, analyser, comprendre) (Paris : PUF)

Mortureux, Marie-Françoise. 1984. «La Dénomination, approche socio-linguistique », Langages $76,95-112$

Mortureux, Marie-Françoise. 1993. « Paradigmes désignationnels », Semen 8, 121-141

Lins Muniz, Elizabeth, et al. (éds). 2010. Dicionário da língua portuguesa (São Paulo : Baixa Planeta)

Pêcheux, Michel. 1990 [1975]. « Les vérités de La Palice. Linguistique, sémantique, philosophie », L'inquiétude du discours (Paris : Éditions des Cendres)

Prefeitura de São Paulo. 2014. Plano Diretor Estratégicoda Cidade de São Paulo [En ligne:http:// www.prefeitura.sp.gov.br/cidade/secretarias/upload/chamadas/2014-07-31_-_lei_16050__plano_diretor_estratgico_1428507821.pdf]

Searle, John R. 1998 [1995]. La construction de la réalité sociale (Paris : Gallimard)

Sériot, Patrick. 1986. «Langue russe et discours politique soviétique : analyse des nominalisations ", Langages 81,11-41

Siblot, Paul. 2001.« De la dénomination à la nomination. Les dynamiques de la signifiance nominale et le propre du nom », Bosredon, Bernard et al. (éds). Linguistique de la dénomination (Cahiers de praxématique 36 ), 189-214

Silva, Maria Laís Pereira da. 2005. Favelas Cariocas : 1930- 1964 (Rio de Janeiro : Contraponto)

Tuma, Romeu. 2003. « Homenagem a Luís Alves de Lima e Silva, o Duque de Caxias, pelo transcurso do seu bicentenário de nascimento », Diário do Senado Federal (Brasilia : 25030) 
Valadares, Lícia do Prado. 2005. A invenção da favela: do mito de origem à favela.com (Rio de Janeiro : FGV)

\section{NOTES}

1. http://g1.globo.com/rio-de-janeiro/rio-450-anos/fotos/2015/02/450-anos-g1-lista-dezcomunidades-visitadas-por-turistas-no-rio.html (17/2/2015)

2. http://www1.folha.uol.com.br/paineldoleitor/2015/03/1610487-usp-clama-por-mudancas-dizleitor-sobre-artigo-de-marco-antonio-zago.shtml (31/3/2015)

3. http://www1.folha.uol.com.br/folha/mundo/ult94u82123.shtml (02/4/2005)

4. http://www1.folha.uol.com.br/livrariadafolha/2014/12/1561017-coletanea-editada-por-tonybellotto-traz-historias-noir-ambientadas-no-rio.shtml (15.12/2014)

5. http://www1.folha.uol.com.br/ambiente/2012/06/1108569-fundacao-ford-diz-que-belo-montepassa-pelo-debate-com-os-indios.shtml (22/6/2012)

6. http://www1.folha.uol.com.br/multimidia/podcasts/784820-novos-baianos-sao-homenageadosnesta-quarta-feira.shtml (18/8/2010)

7. http://www1.folha.uol.com.br/multimidia/tvfolha/2015/06/1643093-reduto-das-farc-reunepobreza-e-beleza-numa-terra-sem-lei-assista.shtml (17/6/2011)

8. http://radioitaperunafm.com/site/2011/11/13/choque-de-paz-rocinha-e-vidigal-saoocupados-por-forcas-de-seguranca (13/11/11)

9. http://www.geocritiq.com/2014/04/as-favelas-apos-a-pacificacao-do-territorio-desafios-ademocratizacao-da-cidade/ (Avril 2014)

10. http://exame.abril.com.br/brasil/noticias/pacificacao-no-rio-completa-5-anos-cercada-porpolemicas, 17/11/13)

11. http://construcaomercado.pini.com.br/negocios-incorporacao-construcao/127/ artigo282612-1.aspx (février 2012)

12. http://www.rj.gov.br (03/11.13, accès via google par «jovens de comunidades apontam benefícios da pacificação »)

13. Voir le site du sénat (senado.gov.br/atividade/pronunciamento), mots-clefs.

14. http://g1.globo.com/politica/processo-de-impeachment-de-dilma/noticia/2015/12/dizerque-impeachment-vai-pacificar-o-pais-e-erro-grosseiro-diz-cardozo.html (7/12/15)

15. http://www1.folha.uol.com.br/poder/2015/12/1716251-dilma-quer-cancelar-folga-paracongresso-votar-o impeachment.shtml

16. http://sao-paulo.estadao.com.br/noticias/geral,novo-reitor-tera-missao-de-pacificarcomunidade-imp-,1096104, (13/11/2013)

17. http://www.viomundo.com.br/politica/depois-de-reduzir-violencia-projeto-das-upps-dasinais-de-fadiga-controle-das-milicias-sobre-os-bairros-pobres-do-rio-se-amplia (11/2/2014)

18. http://www1.folha.uol.com.br/cotidiano/2014/08/1505567-duas-pessoas-morrem-emtiroteios-em-comunidades-pacificadas-no-rio.shtml (25/8/2014) 


\section{RÉSUMÉS}

L'usage qui s'impose aujourd'hui au Brésil est de ne plus parler de favela mais de comunidade(communauté), au point que si les deux termes sont encore mutuellement et significativement substituables, on peut se risquer à prédire que, dans un proche avenir, favela a toutes les chances d'apparaître comme un non-désignant de la réalité que ce terme a été jusqu'à maintenant chargé de désigner. Pourquoi un tel changement? On avance ici l'hypothèse que l'accès de la favela au statut linguistique de comunidade, en l'identifiant idéalement à un ensemble de personnes qui s'auto-contrôlent de l'intérieur, naturalise l'occupation du dehors comme dispositif de préservation de cet idéal communautaire, dès lors que l'auto-contrôle attendu ne s'exerce pas. Après avoir localisé la puissance de la désignation dans l'institution du réel, on éclairera les conditions et les effets de seuil du déploiement discursif de la désignation de comunidade, en lieu et place de favela dans les représentations collectives, avant d'analyser comment l'appropriation discursive, médiatique et sociale du désignant comunidadea introduit et consacré une formule - pacificação da comunidade - qui, en effaçant paradoxalement le trait dominant communautaire au profit $\mathrm{du}$ sème territoire, reconduit potentiellement toute communauté, quelle que soit sa nature, comme un territoire à pacifier.

Today in Brazil the norm is no longer to talk about favela but about comunidade (community), to the point that, if the two terms are still mutually and significantly substitutable, one can venture to predict that, in a near future, favela will appear as a non-designating word of the reality that it was responsible for representing until now. Why has such a change occurred? We advance the hypothesis that the favela's access to the linguistic status of comunidade, by identifying a set of people as a self-controlled domestic community, naturalizes the occupation of this community from outside(rs) in order to preserve this ideal status of community, since the expected selfcontrol is not exercised. After pointing out the power of designation in the institution of a reality, we will shed light on the conditions and threshold effects of the discursive deployment of comunidade instead of favela, in collective representations. Finally, we will analyze how the discursive, social and media spread of the word comunidade introduced and sanctioned the formula - pacificação da comunidade - which, paradoxically erasing the main meaning of community in favor of the secondary meaning of territory, represents potentially every community, regardless of its nature, as a territory which must be pacified..

\section{INDEX}

Mots-clés : catégorisation, comunidade, désignation, favela, pacification

Keywords : categorization, comunidade, designation, favela, pacification

\section{AUTEUR}

\section{PATRICK DAHLET}

Université Fédérale du Minas Gerais (Brésil) 